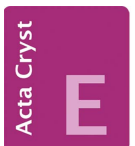

CRYSTALLOGRAPHIC COMMUNICATIONS

ISSN 2056-9890

Received 6 April 2018

Accepted 15 April 2018

Edited by H. Stoeckli-Evans, University of Neuchâtel, Switzerland

Keywords: crystal structure; Schiff base; antioxidant capacity; CUPRAC; hydrogen bonding; $\mathrm{C}-\mathrm{H} \cdots \pi$ interactions; Hirshfeld surface analysis.

CCDC reference: 1837095

Supporting information: this article has supporting information at journals.iucr.org/e

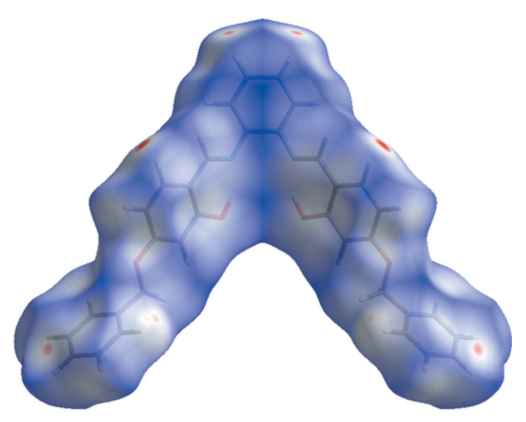

OPEN $\odot$ ACCESS

\section{Crystal structure, Hirshfeld surface analysis and antioxidant capacity of $2,2^{\prime}-\left\{\left(1 E, 1^{\prime} E\right)\right.$-[1,2-phenyl- enebis(azanylylidene)]bis(methanylylidene)\}bis(5- benzyloxy)phenol}

\author{
Nadir Ghichi, ${ }^{\text {a* }}$ Ali Benboudiaf, ${ }^{a}$ Yacine DJebli, ${ }^{b}$ Chawki Bensouici ${ }^{c}$ and Hocine \\ Merazig $^{a}$
} a Unit of Research CHEMS, University of Constantine1, Algeria, ${ }^{\mathbf{b}}$ Laboratory of Materials Chemistry, University of
Constantine1, Algeria, and ${ }^{\mathbf{c}}$ Biotechnology Research Center, Constantine, Algeria. *Correspondence e-mail:
nadirgh82@hotmail.com

The whole molecule of the title Schiff base compound, $\mathrm{C}_{34} \mathrm{H}_{28} \mathrm{~N}_{2} \mathrm{O}_{4}$, is generated by mirror symmetry, with the mirror bisecting the central benzene ring. It was synthesized via the condensation reaction of 1,2-diaminebenzene with 4-benzyloxy-2-hydroxybenzaldehyde. The molecule is $\mathrm{V}$-shaped and there are two intramolecular $\mathrm{O}-\mathrm{H} \cdots \mathrm{N}$ hydrogen bonds present forming $S(6)$ ring motifs. The configuration about the $\mathrm{C}=\mathrm{N}$ imine bonds is $E$. The central benzene ring makes dihedral angles of 41.9 (2) and $43.6(2)^{\circ}$ with the phenol ring and the outer benzyloxy ring, respectively. The latter two rings are inclined to each other by $84.4(2)^{\circ}$. In the crystal, molecules are linked by $\mathrm{C}-\mathrm{H} \cdots \pi$ interactions, forming layers lying parallel to the $a b$ plane. The Hirshfeld surface analysis and the two-dimensional fingerprint plots confirm the predominance of these interactions in the crystal structure. The antioxidant capacity of the compound was determined by the cupric reducing antioxidant capacity (CUPRAC) process.

\section{Chemical context}

Schiff base derivatives are a biologically versatile class of compounds possessing diverse activities, such as anti-oxidant (Haribabu et al., 2015, 2016), anti-inflammatory (Alam et al., 2012), antianxiety, antidepressant (Jubie et al., 2011), antitumour, antibacterial, and fungicidal properties (Refat et al., 2008; Kannan \& Ramesh, 2006). Bis-bidentate Schiff base ligands have been studied extensively and used as building blocks in metallo-supramolecular chemistry (Birkedal \& Pattison, 2006; Shahverdizadeh \& Tiekink, 2011; Chu \& Huang, 2007; Yoshida \& Ichikawa, 1997; Kruger et al., 2001). The common structural feature of these compounds is the presence of an azomethine group, linked by a $\eta$ methylene bridge, which can act as a hydrogen-bond acceptor. In view of this interest we have synthesized the title compound, (I), and report herein on its crystal structure. The ${ }^{1} \mathrm{H}$ NMR NMR spectrum reveals the presence of an imino group $(\mathrm{N}=\mathrm{CH})$ in the range $\delta=8.5-8.7$ p.p.m. The antioxidant capacity of the compound was determined by the cupric reducing antioxidant capacity (CUPRAC) process. 


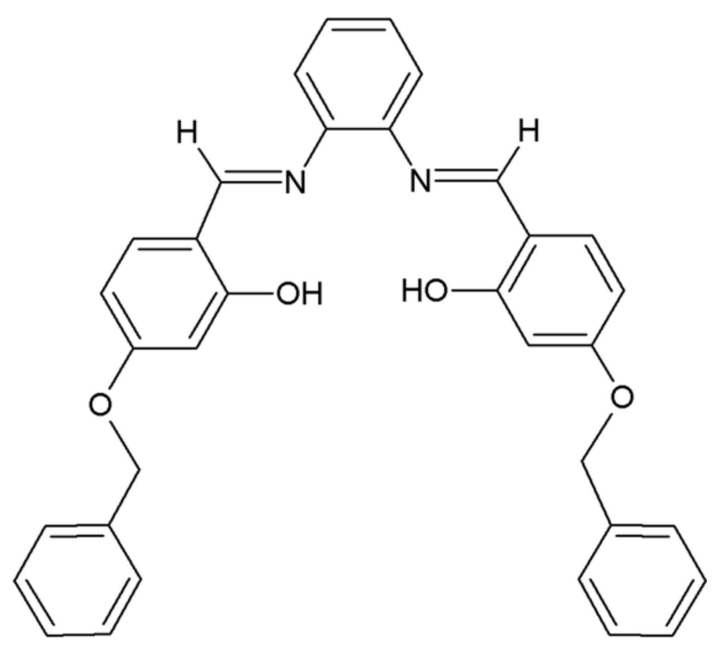

\section{Structural commentary}

The molecular structure of compound (I) is illustrated in Fig. 1. The asymmetric unit consists of half a molecule, with the whole molecule being generated by mirror symmetry. The mirror bisects the central benzene ring, viz. bonds $\mathrm{C} 1-\mathrm{Cl}^{\mathrm{i}}$ and $\mathrm{C} 3-\mathrm{C}^{\mathrm{i}}$ [symmetry code: (i) $-x, y, z$ ]. In the molecule there are two intramolecular $\mathrm{O}-\mathrm{H} \cdots \mathrm{N}$ hydrogen bonds present (Table 1), which form $S(6)$ ring motifs as shown in Fig. 1. The configuration of the $\mathrm{C} 4=\mathrm{N} 1$ imine bonds is $E$ and the $\mathrm{C} 4=\mathrm{N} 1$ bond length is $1.278(6) \AA$. The $\mathrm{C} 3-\mathrm{N} 1=\mathrm{C} 4$ bond angles are less than $120^{\circ}\left[118.9(4)^{\circ}\right]$, and the imine group has a $\mathrm{C} 3-\mathrm{N} 1-\mathrm{C} 4-\mathrm{C} 5$ torsion angle of $-176.8(4)^{\circ}$. The molecule is $\mathrm{V}$-shaped and the two arms are non-planar; the central benzene ring forms dihedral angles of 41.9 (2) and

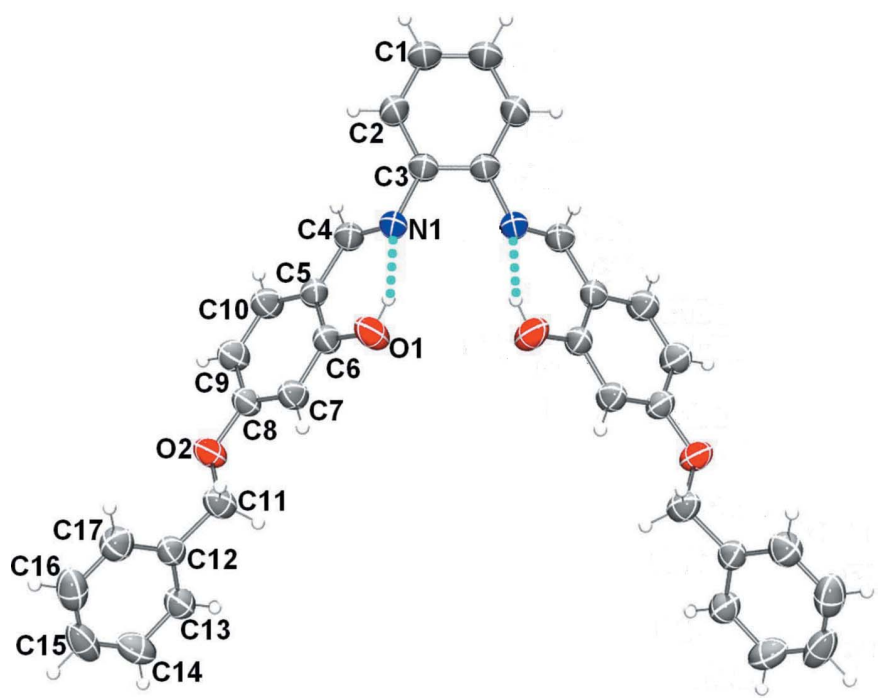

Figure 1

View of the molecular structure of compound (I), with atom labelling. Displacement ellipsoids are drawn at the $50 \%$ probability level. Unlabelled atoms are related to labelled atoms by the mirror symmetry code: (i) $-x, y, z$. The intramolecular $\mathrm{O}-\mathrm{H} \cdots \mathrm{N}$ hydrogen bonds (see Table 1) are shown as dashed lines.
Table 1

Hydrogen-bond geometry $\left(\AA{ }^{\circ}\right)$.

$\mathrm{Cg} 2$ is the centroid of the $\mathrm{C} 5-\mathrm{C} 10$ phenol ring.

\begin{tabular}{lllll}
\hline$D-\mathrm{H} \cdots A$ & $D-\mathrm{H}$ & $\mathrm{H} \cdots A$ & $D \cdots A$ & $D-\mathrm{H} \cdots A$ \\
\hline $\mathrm{O} 1-\mathrm{H} 1 O \cdots \mathrm{N} 1$ & 0.82 & 1.90 & $2.622(5)$ & 147 \\
$\mathrm{C} 2-\mathrm{H} 2 \cdots C g 2^{\mathrm{i}}$ & 0.93 & 2.88 & $3.499(5)$ & 125 \\
$\mathrm{C} 13-\mathrm{H} 13 \cdots C g 2^{\mathrm{ii}}$ & 0.93 & 2.60 & $3.493(5)$ & 161 \\
\hline
\end{tabular}

Symmetry codes: (i) $x,-y+1, z-\frac{1}{2}$; (ii) $x,-y, z+\frac{1}{2}$.

$43.6(2)^{\circ}$ with the phenol ring (C5-C10) and the outer benzyloxy ring (C12-C17), respectively. The latter two rings are almost normal to each other, with a dihedral angle of $84.4(2)^{\circ}$.

\section{Supramolecular features and Hirshfeld surface analysis}

In the crystal of (I), molecules are linked by $\mathrm{C}-\mathrm{H} \cdots \pi$ interactions (Table 1), forming layers parallel to the (001) plane, as illustrated in Fig. 2.

The Hirshfeld surface analysis (Spackman \& Jayatilaka, 2009) and the associated two-dimensional fingerprint plots (McKinnon et al., 2007) were performed with CrystalExplorer17 (Turner et al., 2017). The Hirshfeld surface of compound (I) mapped over $d_{\text {norm }}$ is given in Fig. 3, and the fingerprint plots are given in Fig. 4. They reveal that the principal intermolecular interactions are $\mathrm{H} \cdots \mathrm{H}$ at $45.7 \%$ (Fig. $4 b$ ) and $\mathrm{H} \cdots \mathrm{C} / \mathrm{C} \cdots \mathrm{H}$ at $34.6 \%$ (Fig. $4 c$ ), followed by the $\mathrm{H} \cdots \mathrm{O} / \mathrm{O} \cdots \mathrm{H}$ interactions at $13.6 \%$ (Fig. 4 ).

\section{Database survey}

A search of the Cambridge Structural Database (CSD, Version 5.39, last update February 2018; Groom et al., 2016) for similar compounds yielded four hits. These compounds (see Fig. 5) include 5,5'-dihydroxy-2,2'-[o-phenylenebis(nitrilomethylene)]diphenol ethanol solvate (II) (CSD refcode HUVXUT; Soroceanu et al., 2013), 5,5'-dimethoxy2,2'-[4,5-dimethyl-o-phenylenebis(nitrilomethylidyne)]di-

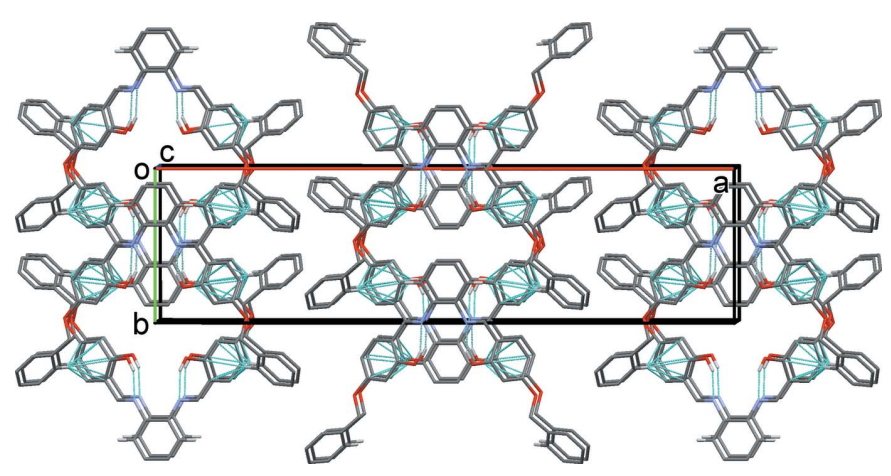

Figure 2

Crystal packing of compound (I) viewed along the $c$ axis, with the $\mathrm{O}-$ $\mathrm{H} \cdots \mathrm{N}$ intramolecular hydrogen bonds and the $\mathrm{C}-\mathrm{H} \cdots \pi$ interactions (see Table 1) illustrated as dashed lines. 


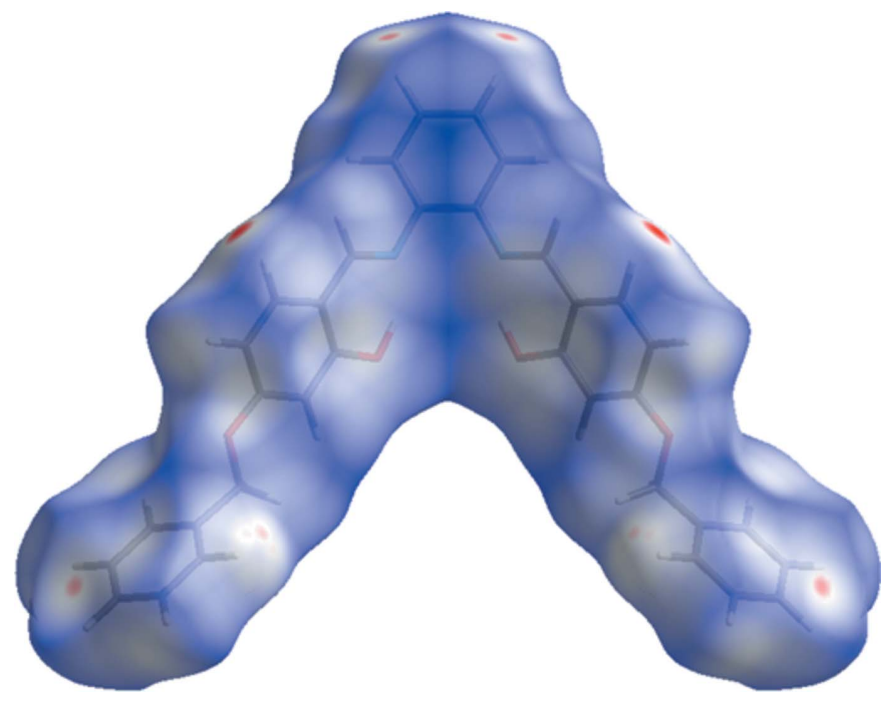

Figure 3

View of the Hirshfeld surface of (I) mapped over $d_{\text {norm }}$.

phenol (III) (KUSJIS; Kargar et al., 2010), 1,2-bis\{[(2-hydroxy-4-methoxyphenyl)(phenyl)methylene]amino\}benzene (IV) (SOXCIS; Lippe et al., 2009) and 5,5'-dimethoxy-2,2'-1,2phenylenebis(nitrilomethylidyne)]diphenol (V) (XIFREK; Eltayeb et al., 2007). In all four compounds there are two intramolecular $\mathrm{O}-\mathrm{H} \cdots \mathrm{N}$ hydrogen bonds present forming $S(6)$ ring motifs.

In (II) the phenol rings are inclined to the central benzene ring by 53.9 (3) and $4.0(2)^{\circ}$ and to each other by $49.9(2)^{\circ}$. In (III) the corresponding dihedral angles are 48.12 (8), 21.44 (8) and $47.70(8)^{\circ}$, while in $(\mathrm{V})$ the corresponding dihedral angles
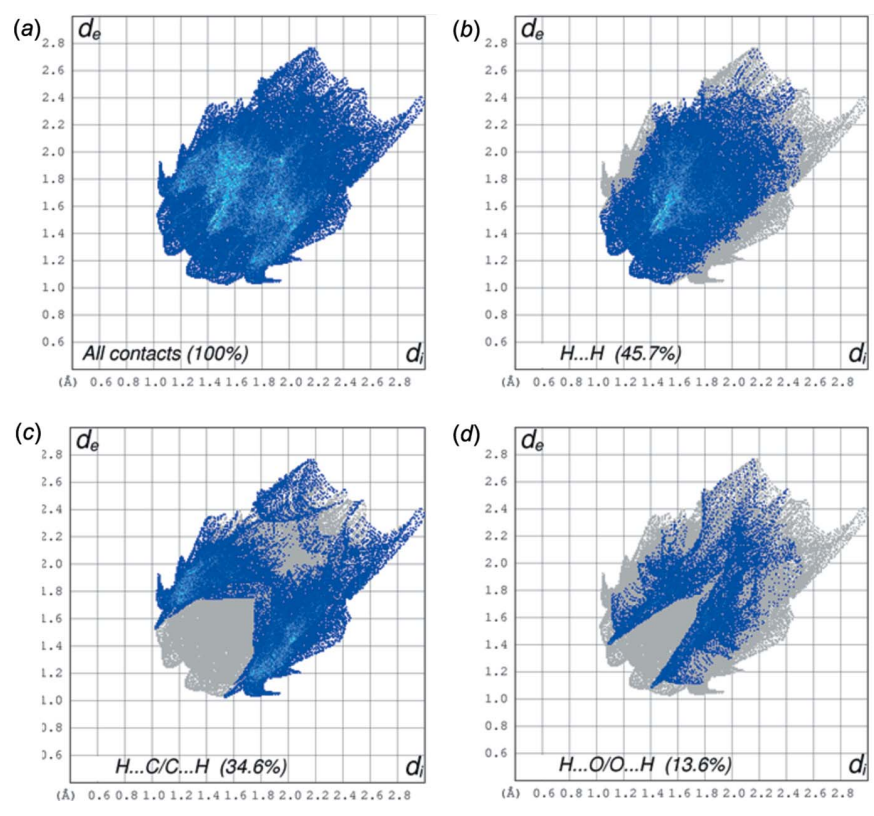

Figure 4

The two-dimensional fingerprint plots of $(\mathrm{I}):(a)$ all interactions; $(b)$ $\mathrm{H} \cdots \mathrm{H} ;(c) \mathrm{H} \cdots \mathrm{C} / \mathrm{C} \cdots \mathrm{H} ;(d) \mathrm{H} \cdots \mathrm{O} / \mathrm{O} \cdots \mathrm{H}$.
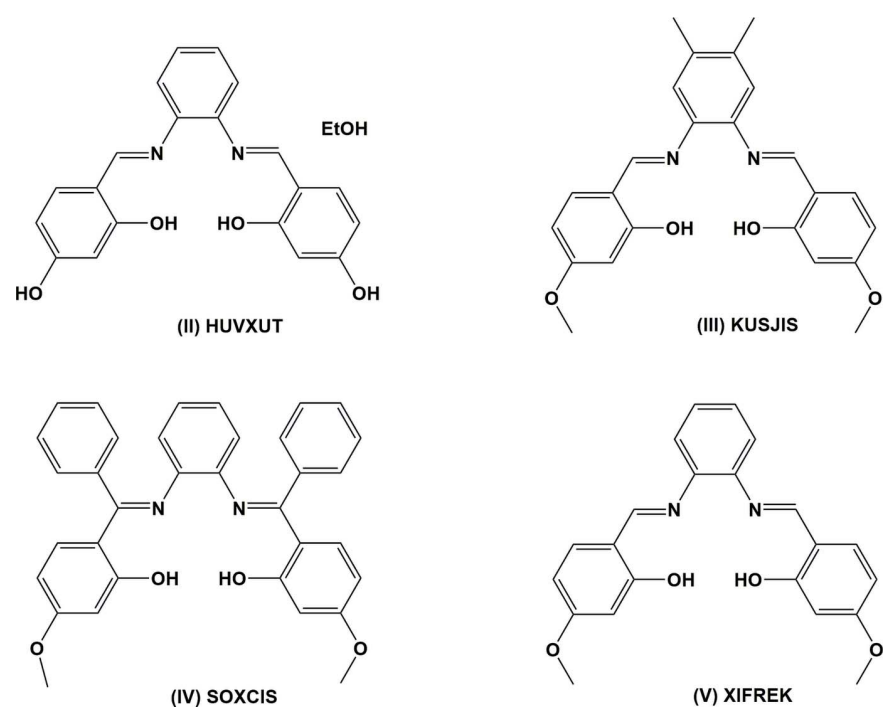

Figure 5

Similar compounds to that of the title compound, (I), in the CSD; see Section 4, Database survey.

are $58.29(12), 2.20(12)$ and $57.60(12)^{\circ}$. In compound (IV), that possesses twofold rotational symmetry with the twofold axis bisecting the central benzene ring, the phenol rings are inclined to the central benzene ring by $82.30(5)^{\circ}$ and to each other by $63.76(5)^{\circ}$. In the title compound, which possesses mirror symmetry, the corresponding dihedral angles are $41.9(2)$ and $68.9(2)^{\circ}$.

A search of the CSD for metal complexes of compounds similar to compound (I) gave over 30 hits. The ligands always coordinate in a tetradentate manner. For example, there were 13 hits for transition metal complexes of compound (II). The majority involve square-planar coordinated metal atoms, such as in complexes (5,5'-dihydroxy-2,2'-[o-phenylenebis(nitrilomethylidyne)]diphenolato)nickel(II) dihydrate (POFFOG; Fun et al., 2008) and (4,4'-\{1,2-phenylenebis[(nitrilo- $\kappa N)$ methylylidene]\}dibenzene-1,3-diolato- $\kappa O^{3}$ )copper(II)

methanol solvate (DUQBEX; Niu et al., 2010). For compound (V), five hits were found; they include three sixfold-coordinated tin complexes (DOSCOF, DOSDAS, DOSFOI; MuñozFlores et al., 2014) and two square-pyramidal manganese complexes (ODESEY, Ghaemi et al., 2016; XIYQOM, Eltayeb et al., 2008).

\section{Antioxidant activity}

The antioxidant activity profile of the synthesized compound (I) was determined by utilizing the copper(II)-neocuprine [Cu $\left.{ }^{\mathrm{II}}-\mathrm{Nc}\right]$ (CUPRAC) method (Apak et al., 2004). The CUPRAC method (Fig. 6) (cupric ion reducing antioxidant capacity) is based on the follow-up of the decrease in the increased absorbance of the neocuproene (Nc), copper $\left(\mathrm{Cu}^{+2}\right) \mathrm{Nc}_{2}-\mathrm{Cu}^{+2}$ complex. Indeed, in the presence of an antioxidant agent, the copper-neocuproene complex is reduced and this reaction is quantified spectrophotometrically at a wavelength of $450 \mathrm{~nm}$. 
Table 2

Cupric ion reducing antioxidant capacity of compound (I).

\begin{tabular}{|c|c|c|c|c|c|c|c|c|}
\hline & \multicolumn{8}{|c|}{ Percentage (\%) Inhibition } \\
\hline & $12.5 \mu \mathrm{g}$ & $25 \mu \mathrm{g}$ & $50 \mu \mathrm{g}$ & $100 \mu \mathrm{g}$ & $200 \mu \mathrm{g}$ & $400 \mu \mathrm{g}$ & $800 \mu \mathrm{g}$ & $\mathrm{A} 0.50\left(\mu \mathrm{g} \mathrm{ml}^{-1}\right)$ \\
\hline Compound (I) & $0.39 \pm 0.01$ & $0.59 \pm 0.01$ & $0.91 \pm 0.03$ & $1.42 \pm 0.02$ & $1.84 \pm 0.36$ & $3.12 \pm 0.25$ & $4.29 \pm 0.11$ & $15.03 \pm 1.50$ \\
\hline BHT & $1.41 \pm 0.03$ & $2.22 \pm 0.05$ & $2.42 \pm 0.02$ & $2.50 \pm 0.01$ & $2.56 \pm 0.05$ & $2.86 \pm 0.07$ & $3.38 \pm 0.13$ & $8.97 \pm 3.94$ \\
\hline
\end{tabular}

According to the cupric ion reducing antioxidant capacity assay, the title compound displayed activity with variable potency in all tested concentrations, because the percentage (\%) inhibition in the CUPRAC assay is good $\left[\mathrm{A}_{0.50}=15.03 \pm\right.$ 1.50 for a $4 \mathrm{mg}$ dosage, compared to the results for buthylated toluene (BHT) $\left[\mathrm{A}_{0.50}=8.97 \pm 3.94\right]$, used as a positive control (see Table 2). Note: In CUPRAC antioxidant activity, the values expressed are the mean \pm s.u.s of three parallel measurements $(p<0.05)$.

\section{Synthesis and crystallization}

1,2-Diaminebenzene $(0.027 \mathrm{~g})$ and 4-benzyloxy-2-hydroxybenzaldehyde $(0.1141 \mathrm{~g})$ in ethanol $(15 \mathrm{ml})$ were refluxed for $1 \mathrm{~h}$, then the solvent was evaporated in vacuo. The residue was recrystallized from ethanol, yielding yellow block-like crystals of the title compound on slow evaporation of the solvent. The purity of the compound was characterized by its NMR spectrum $\left(250 \mathrm{MHz}, \mathrm{CDCl}_{3}\right)$. The azomethine proton appears in the 8.5-8.7 p.p.m. range, while the imine bond is characterized in the ${ }^{13} \mathrm{C}$ RMN spectrum with the imine $\mathrm{C}$ and $\mathrm{OH}$ signals in the range 162.23-163.34 p.p.m. ${ }^{1} \mathrm{H}$ NMR: $\delta=6.5-7.6(\mathrm{~m}, 12 \mathrm{H}$; $H$-ar $), \delta=13.7(s, 1 \mathrm{H} ; \mathrm{OH}), \delta=5.1\left(\mathrm{~s}, 1 \mathrm{H} ; \mathrm{CH}_{2}-\mathrm{O}\right) .{ }^{13} \mathrm{C}$ NMR: $70.15,120.33,127.30,127.64,128.26,128.75,142.32,162.23$, $163.33,163.34$.

\section{Refinement}

Crystal data, data collection and structure refinement details are summarized in Table 3. The hydroxyl $\mathrm{H}$ atom was located in a difference-Fourier map and initially freely refined. In the final cycles of refinements it was positioned geometrically $(\mathrm{O}-\mathrm{H}=0.82 \AA)$ and refined as riding with $U_{\text {iso }}(\mathrm{H})=$

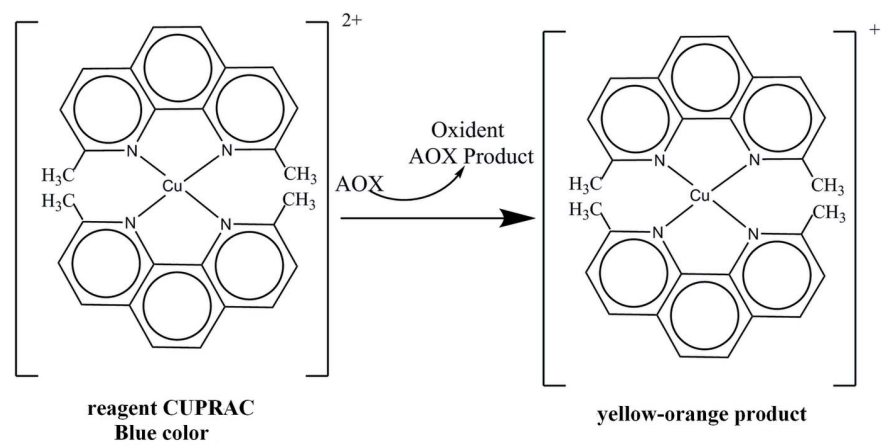

Figure 6

Reduction of the chromogenic complex of $\mathrm{Cu}^{+2}-\mathrm{Nc}$
$1.5 U_{\text {eq }}(\mathrm{O})$. The $\mathrm{C}$-bound $\mathrm{H}$ atoms were positioned geometrically $(\mathrm{C}-\mathrm{H}=0.93-0.97 \AA)$ and refined as riding with $U_{\text {iso }}(\mathrm{H})$ $=1.2 U_{\text {eq }}(\mathrm{C})$.

\section{Funding information}

We are grateful to the Department of Higher Scientific Research and CHEMS Research Unit, University of Constantine1, Algeria, for funding this research project.

\section{References}

Alam, M. S., Choi, J.-H. \& Lee, D.-U. (2012). Bioorg. Med. Chem. 20, 4103-4108.

Apak, R., Güçlü, K., Özyürek, M. \& Karademir, S. E. (2004). J. Agric. Food Chem. 52, 7970-7981.

Birkedal, H. \& Pattison, P. (2006). Acta Cryst. C62, o139-o141.

Bruker (2011). APEX2 and SAINT. Bruker AXS Inc., Madison, Wisconsion, USA.

Chu, Z. \& Huang, W. (2007). J. Mol. Struct. 837, 15-22.

Eltayeb, N. E., Teoh, S. G., Chantrapromma, S., Fun, H.-K. \& Adnan, R. (2008). Acta Cryst. E64, m670-m671.

Eltayeb, N. E., Teoh, S. G., Chantrapromma, S., Fun, H.-K. \& Ibrahim, K. (2007). Acta Cryst. E63, o3094-03095.

Table 3

Experimental details

\begin{tabular}{ll}
\hline Crystal data & \\
Chemical formula & $\mathrm{C}_{34} \mathrm{H}_{28} \mathrm{~N}_{2} \mathrm{O}_{4}$ \\
$M_{\mathrm{r}}$ & 528.58 \\
Crystal system, space group & Orthorhombic, $C m c 2_{1}$ \\
Temperature $(\mathrm{K})$ & 293 \\
$\begin{array}{l}a, b, c(\AA) \\
V\left(\AA^{3}\right)\end{array}$ & $35.297(3), 9.3902(6), 8.3603(5)$ \\
$Z$ & $2771.0(3)$ \\
Radiation type & 4 \\
$\mu\left(\mathrm{mm}^{-1}\right)$ & Mo $K \alpha$ \\
Crystal size $(\mathrm{mm})$ & 0.08 \\
& $0.03 \times 0.02 \times 0.01$ \\
Data collection & \\
Diffractometer & \\
No. of measured, independent and & Bruker APEXII CCD \\
$\quad$ observed $[I>2 \sigma(I)]$ reflections & $4493,2516,1691$ \\
$R_{\text {int }}$ & \\
$(\text { sin } \theta / \lambda)_{\text {max }}\left(\AA^{-1}\right)$ & 0.042 \\
& 0.651 \\
Refinement & \\
$R\left[F^{2}>2 \sigma\left(F^{2}\right)\right], w R\left(F^{2}\right), S$ & \\
No. of reflections & $0.053,0.158,1.02$ \\
No. of parameters & 2516 \\
No. of restraints & 185 \\
H-atom treatment & 1 \\
$\Delta \rho_{\text {max }}, \Delta \rho_{\text {min }}\left(\mathrm{e} \AA^{-3}\right)$ & H-atom parameters constrained \\
\hline & $0.29,-0.24$ \\
\hline
\end{tabular}

Computer programs: APEX2 and SAINT (Bruker, 2011), SHELXT2017 (Sheldrick, 2015a), SHELXL2017 (Sheldrick, 2015b), SHELXTL (Sheldrick, 2008), Mercury (Macrae et al.2008) and PLATON (Spek, 2009). 
Fun, H.-K., Kia, R., Mirkhani, V. \& Zargoshi, H. (2008). Acta Cryst. E64, m1181-m1182.

Ghaemi, A., Keyvani, B., Rayati, S., Zarei, S. \& Notash, B. (2016). Zh. Strukt. Khim. (Russ. J. Struct. Chem.), 57, 1027-1030.

Groom, C. R., Bruno, I. J., Lightfoot, M. P. \& Ward, S. C. (2016). Acta Cryst. B72, 171-179.

Haribabu, J., Subhashree, G. R., Saranya, S., Gomathi, K., Karvembu, R. \& Gayathri, D. (2015). J. Mol. Struct. 1094, 281-291.

Haribabu, J., Subhashree, G. R., Saranya, S., Gomathi, K., Karvembu, R. \& Gayathri, D. (2016). J. Mol. Struct. 1110, 185-195.

Jubie, S., Sikdar, P., Antony, S., Kalirajan, R., Gowramma, B., Gomathy, S. \& Elango, K. (2011). Pak. J. Pharm. Sci. 24, 109-112.

Kannan, M. \& Ramesh, R. (2006). Polyhedron, 25, 3095-3103.

Kargar, H., Kia, R., Khan, I. U., Sahraei, A. \& Aberoomand Azar, P. (2010). Acta Cryst. E66, o728.

Kruger, P. E., Martin, N. \& Nieuwenhuyzen, M. (2001). J. Chem. Soc. Dalton Trans. pp. 1966-1970.

Lippe, K., Gerlach, D., Kroke, E. \& Wagler, J. (2009). Organometallics, 28, 621-629.

Macrae, C. F., Bruno, I. J., Chisholm, J. A., Edgington, P. R., McCabe, P., Pidcock, E., Rodriguez-Monge, L., Taylor, R., van de Streek, J. \& Wood, P. A. (2008). J. Appl. Cryst. 41, 466-470.

McKinnon, J. J., Jayatilaka, D. \& Spackman, M. A. (2007). Chem. Commun. pp. 3814-3816.
Muñoz-Flores, B. M., Santillán, R., Farfán, N., Álvarez-Venicio, V., Jiménez-Pérez, V. M., Rodríguez, M., Morales-Saavedra, O. G., Lacroix, P. G., Lepetit, C. \& Nakatani, K. (2014). J. Organomet. Chem. 769, 64-71.

Niu, M., Fan, S., Liu, K., Cao, Z. \& Wang, D. (2010). Acta Cryst. E66, $\mathrm{m} 77$.

Refat, M. S., El-Korashy, S. A., Kumar, D. N. \& Ahmed, A. S. (2008). Spectrochim. Acta Part A, 70, 898-906.

Shahverdizadeh, G. H. \& Tiekink, E. R. T. (2011). Acta Cryst. E67, o798.

Sheldrick, G. M. (2008). Acta Cryst. A64, 112-122.

Sheldrick, G. M. (2015a). Acta Cryst. A71, 3-8.

Sheldrick, G. M. (2015b). Acta Cryst. C71, 3-8.

Soroceanu, A., Shova, S., Cazacu, M., Balan, I., Gorinchoy, N. \& Turta, C. (2013). J. Chem. Crystallogr. 43, 310-318.

Spackman, M. A. \& Jayatilaka, D. (2009). CrystEngComm, 11, 1932.

Spek, A. L. (2009). Acta Cryst. D65, 148-155.

Turner, M. J., McKinnon, J. J., Wolff, S. K., Grimwood, D. J., Spackman, P. R., Jayatilaka, D. \& Spackman, M. A. (2017). CrystalExplorer17. University of Western Australia. http://hirshfeldsurface.net

Yoshida, N. \& Ichikawa, K. (1997). Chem. Commun. pp. 1091-1092. 


\section{supporting information}

Acta Cryst. (2018). E74, 682-686 [https://doi.org/10.1107/S2056989018005832]

Crystal structure, Hirshfeld surface analysis and antioxidant capacity of 2,2'-\{(1E,1'E)-[1,2-phenylenebis(azanylylidene)]bis(methanylylidene)\}bis(5benzyloxy)phenol

Nadir Ghichi, Ali Benboudiaf, Yacine DJebli, Chawki Bensouici and Hocine Merazig

Computing details

Data collection: APEX2 (Bruker, 2011); cell refinement: SAINT (Bruker, 2011); data reduction: SAINT (Bruker, 2011); program(s) used to solve structure: SHELXT2017 (Sheldrick, 2015a); program(s) used to refine structure: SHELXL2017 (Sheldrick, 2015b); molecular graphics: SHELXTL (Sheldrick, 2008) and Mercury (Macrae et al., 2008); software used to prepare material for publication: SHELXTL (Sheldrick, 2008) and PLATON (Spek, 2009).

$2,2^{\prime}-\left\{\left(1 E, 1^{\prime} E\right)\right.$-[1,2-Phenylenebis(azanylylidene)]bis(methanylylidene)\}bis(5-benzyloxy) phenol

Crystal data

$\mathrm{C}_{34} \mathrm{H}_{28} \mathrm{~N}_{2} \mathrm{O}_{4}$

$M_{r}=528.58$

Orthorhombic, $\mathrm{Cmc} 2_{1}$

Hall symbol: C 2c -2

$a=35.297(3) \AA$

$b=9.3902(6) \AA$

$c=8.3603(5) \AA$

$V=2771.0(3) \AA^{3}$

$Z=4$

Data collection

Bruker APEXII CCD diffractometer

Detector resolution: 18.4 pixels $\mathrm{mm}^{-1}$

$\varphi$ and $\omega$ scans

4493 measured reflections

2516 independent reflections

Refinement

Refinement on $F^{2}$

Least-squares matrix: full

$R\left[F^{2}>2 \sigma\left(F^{2}\right)\right]=0.053$

$w R\left(F^{2}\right)=0.158$

$S=1.01$

2516 reflections

185 parameters

1 restraint

Primary atom site location: structure-invariant direct methods
$F(000)=1112$

$D_{\mathrm{x}}=1.267 \mathrm{Mg} \mathrm{m}^{-3}$

Mo $K \alpha$ radiation, $\lambda=0.71073 \AA$

Cell parameters from 1621 reflections

$\theta=2.2-21.3^{\circ}$

$\mu=0.08 \mathrm{~mm}^{-1}$

$T=293 \mathrm{~K}$

Block, yellow

$0.03 \times 0.02 \times 0.01 \mathrm{~mm}$

1691 reflections with $I>2 \sigma(I)$

$R_{\text {int }}=0.042$

$\theta_{\max }=27.5^{\circ}, \theta_{\min }=3.7^{\circ}$

$h=-45 \rightarrow 40$

$k=-12 \rightarrow 5$

$l=-10 \rightarrow 6$

Secondary atom site location: difference Fourier map

Hydrogen site location: mixed

$\mathrm{H}$-atom parameters constrained

$w=1 /\left[\sigma^{2}\left(F_{\mathrm{o}}^{2}\right)+(0.0817 P)^{2}\right]$

where $P=\left(F_{\mathrm{o}}{ }^{2}+2 F_{\mathrm{c}}{ }^{2}\right) / 3$

$(\Delta / \sigma)_{\max }<0.001$

$\Delta \rho_{\max }=0.29 \mathrm{e} \AA^{-3}$

$\Delta \rho_{\min }=-0.24$ e $\AA^{-3}$ 


\section{Special details}

Geometry. Bond distances, angles etc. have been calculated using the rounded fractional coordinates. All su's are estimated from the variances of the (full) variance-covariance matrix. The cell esds are taken into account in the estimation of distances, angles and torsion angles

Fractional atomic coordinates and isotropic or equivalent isotropic displacement parameters $\left(\AA^{2}\right)$

\begin{tabular}{|c|c|c|c|c|}
\hline & $x$ & $y$ & $z$ & $U_{\text {iso }} * / U_{\text {eq }}$ \\
\hline $\mathrm{O} 1$ & $0.05052(9)$ & 0.2350 & $0.3234(5)$ & 0.0693 (13) \\
\hline $\mathrm{O} 2$ & $0.15676(8)$ & 0.0288 & $0.5919(4)$ & $0.0546(9)$ \\
\hline N1 & $0.03881(9)$ & $0.5084(4)$ & $0.3640(5)$ & $0.0487(10)$ \\
\hline $\mathrm{C} 1$ & 0.01955 (13) & $0.8879(4)$ & $0.2654(7)$ & $0.0639(15)$ \\
\hline $\mathrm{C} 2$ & $0.03890(12)$ & $0.7645(4)$ & $0.2985(6)$ & 0.0567 (14) \\
\hline $\mathrm{C} 3$ & 0.01982 (11) & $0.6379(4)$ & $0.3322(5)$ & $0.0481(11)$ \\
\hline $\mathrm{C} 4$ & $0.06841(12)$ & $0.5103(4)$ & $0.4519(6)$ & 0.0495 (14) \\
\hline $\mathrm{C} 5$ & 0.09057 (11) & $0.3828(4)$ & $0.4833(5)$ & $0.0454(11)$ \\
\hline C6 & $0.08042(11)$ & $0.2506(4)$ & $0.4207(5)$ & $0.0475(11)$ \\
\hline $\mathrm{C} 7$ & 0.10168 (11) & $0.1285(4)$ & $0.4566(5)$ & 0.0488 (11) \\
\hline $\mathrm{C} 8$ & $0.13349(11)$ & $0.1401(4)$ & $0.5523(5)$ & $0.0463(12)$ \\
\hline $\mathrm{C} 9$ & $0.14420(12)$ & $0.2710(4)$ & $0.6146(6)$ & $0.0560(16)$ \\
\hline $\mathrm{C} 10$ & $0.12269(12)$ & $0.3889(4)$ & $0.5813(5)$ & $0.0560(16)$ \\
\hline C11 & $0.14781(12)$ & $-0.1086(4)$ & $0.5260(6)$ & 0.0547 (16) \\
\hline $\mathrm{C} 12$ & $0.17886(11)$ & $-0.2104(4)$ & $0.5695(5)$ & $0.0449(11)$ \\
\hline $\mathrm{C} 13$ & $0.17544(12)$ & $-0.2993(4)$ & $0.6998(6)$ & $0.0543(16)$ \\
\hline $\mathrm{C} 14$ & $0.20330(16)$ & $-0.3988(4)$ & $0.7345(6)$ & 0.0677 (17) \\
\hline $\mathrm{C} 15$ & $0.23502(14)$ & $-0.4070(5)$ & $0.6404(7)$ & 0.0700 (19) \\
\hline $\mathrm{C} 16$ & $0.23920(14)$ & $-0.3165(6)$ & $0.5124(7)$ & 0.0697 (17) \\
\hline C17 & $0.21118(13)$ & $-0.2190(5)$ & $0.4765(6)$ & $0.0617(17)$ \\
\hline H1 & 0.03276 & 0.97121 & 0.24301 & $0.0770 *$ \\
\hline $\mathrm{H} 1 \mathrm{O}$ & 0.04038 & 0.31254 & 0.30933 & $0.1040^{*}$ \\
\hline $\mathrm{H} 2$ & 0.06524 & 0.76529 & 0.29851 & $0.0680^{*}$ \\
\hline H4 & $0.0777(10)$ & $0.604(4)$ & $0.509(5)$ & $0.041(9)^{*}$ \\
\hline $\mathrm{H} 7$ & 0.09442 & 0.04030 & 0.41635 & $0.0580^{*}$ \\
\hline H9 & 0.16571 & 0.27872 & 0.67824 & $0.0670^{*}$ \\
\hline H10 & 0.12968 & 0.47594 & 0.62529 & $0.0670^{*}$ \\
\hline $\mathrm{H} 11 \mathrm{~A}$ & 0.12385 & -0.14214 & 0.56862 & $0.0660 *$ \\
\hline H11B & 0.14557 & -0.10203 & 0.41061 & $0.0660 *$ \\
\hline H13 & 0.15420 & -0.29259 & 0.76516 & $0.0650 *$ \\
\hline H14 & 0.20047 & -0.45974 & 0.82141 & $0.0810^{*}$ \\
\hline H15 & 0.25371 & -0.47378 & 0.66311 & $0.0840 *$ \\
\hline H16 & 0.26095 & -0.32099 & 0.44979 & $0.0840 *$ \\
\hline H17 & 0.21407 & -0.15860 & 0.38915 & $0.0740^{*}$ \\
\hline
\end{tabular}

Atomic displacement parameters $\left(\AA^{2}\right)$

\begin{tabular}{lllllll}
\hline & $U^{11}$ & $U^{22}$ & $U^{33}$ & $U^{12}$ & $U^{13}$ & $U^{23}$ \\
\hline O1 & $0.0640(19)$ & $0.0400(16)$ & $0.104(3)$ & $0.0056(14)$ & $-0.0359(19)$ & $-0.0063(17)$ \\
O2 & $0.0595(17)$ & $0.0386(14)$ & $0.0658(17)$ & $0.0080(12)$ & $-0.0166(15)$ & $-0.0028(14)$
\end{tabular}




$\begin{array}{lllllll}\mathrm{N} 1 & 0.0416(17) & 0.0335(16) & 0.071(2) & 0.0021(14) & 0.0019(18) & 0.0024(15) \\ \mathrm{C} 1 & 0.063(3) & 0.0317(19) & 0.097(3) & -0.0042(16) & -0.001(3) & 0.003(2) \\ \mathrm{C} 2 & 0.047(2) & 0.041(2) & 0.082(3) & -0.0048(17) & -0.002(2) & 0.000(2) \\ \mathrm{C} 3 & 0.049(2) & 0.0322(18) & 0.063(2) & 0.0022(16) & 0.002(2) & -0.0028(17) \\ \mathrm{C} 4 & 0.049(2) & 0.0345(19) & 0.065(3) & 0.0002(17) & 0.003(2) & -0.0024(18) \\ \mathrm{C} 5 & 0.042(2) & 0.0373(19) & 0.057(2) & -0.0015(16) & 0.002(2) & -0.0033(18) \\ \mathrm{C} 6 & 0.042(2) & 0.0364(19) & 0.064(2) & -0.0017(16) & -0.008(2) & -0.0015(17) \\ \text { C7 } & 0.051(2) & 0.0365(18) & 0.059(2) & -0.0007(16) & -0.010(2) & -0.0041(19) \\ \text { C8 } & 0.047(2) & 0.039(2) & 0.053(2) & 0.0063(16) & -0.0047(19) & 0.0005(17) \\ \text { C9 } & 0.058(3) & 0.045(2) & 0.065(3) & -0.0010(18) & -0.017(2) & -0.003(2) \\ \text { C10 } & 0.060(3) & 0.039(2) & 0.069(3) & -0.0035(18) & -0.011(2) & -0.006(2) \\ \text { C11 } & 0.059(3) & 0.041(2) & 0.064(3) & 0.0043(19) & -0.013(2) & -0.0047(19) \\ \text { C12 } & 0.043(2) & 0.0378(19) & 0.054(2) & 0.0001(16) & -0.0069(18) & -0.0056(18) \\ \text { C13 } & 0.054(3) & 0.049(2) & 0.060(3) & 0.0042(18) & -0.004(2) & -0.001(2) \\ \text { C14 } & 0.082(3) & 0.051(3) & 0.070(3) & 0.013(2) & -0.021(3) & 0.005(2) \\ \text { C15 } & 0.060(3) & 0.055(3) & 0.095(4) & 0.020(2) & -0.023(3) & -0.028(3) \\ \text { C16 } & 0.053(3) & 0.075(3) & 0.081(3) & 0.006(2) & 0.001(3) & -0.021(3) \\ \text { C17 } & 0.062(3) & 0.057(3) & 0.066(3) & -0.005(2) & -0.002(2) & -0.001(2) \\ & & & & & & \end{array}$

Geometric parameters $\left(\AA,{ }^{\circ}\right)$

\begin{tabular}{|c|c|c|c|}
\hline $\mathrm{O} 1-\mathrm{C} 6$ & $1.341(5)$ & $\mathrm{C} 12-\mathrm{C} 13$ & $1.378(6)$ \\
\hline $\mathrm{O} 2-\mathrm{C} 8$ & $1.370(5)$ & $\mathrm{C} 13-\mathrm{C} 14$ & $1.387(6)$ \\
\hline $\mathrm{O} 2-\mathrm{C} 11$ & $1.438(5)$ & $\mathrm{C} 14-\mathrm{C} 15$ & $1.371(8)$ \\
\hline $\mathrm{N} 1-\mathrm{C} 3$ & $1.414(5)$ & $\mathrm{C} 15-\mathrm{C} 16$ & $1.374(8)$ \\
\hline $\mathrm{N} 1-\mathrm{C} 4$ & $1.278(6)$ & $\mathrm{C} 16-\mathrm{C} 17$ & $1.381(7)$ \\
\hline $\mathrm{O} 1-\mathrm{H} 1 \mathrm{O}$ & 0.8200 & $\mathrm{C} 1-\mathrm{H} 1$ & 0.9300 \\
\hline $\mathrm{C} 1-\mathrm{C} 1^{\mathrm{i}}$ & $1.380(6)$ & $\mathrm{C} 2-\mathrm{H} 2$ & 0.9300 \\
\hline $\mathrm{C} 1-\mathrm{C} 2$ & $1.373(6)$ & $\mathrm{C} 4-\mathrm{H} 4$ & $1.05(4)$ \\
\hline $\mathrm{C} 2-\mathrm{C} 3$ & $1.395(5)$ & $\mathrm{C} 7-\mathrm{H} 7$ & 0.9300 \\
\hline $\mathrm{C} 3-\mathrm{C} 3^{\mathrm{i}}$ & $1.399(5)$ & C9- $\mathrm{H} 9$ & 0.9300 \\
\hline $\mathrm{C} 4-\mathrm{C} 5$ & $1.454(5)$ & $\mathrm{C} 10-\mathrm{H} 10$ & 0.9300 \\
\hline $\mathrm{C} 5-\mathrm{C} 10$ & $1.400(6)$ & $\mathrm{C} 11-\mathrm{H} 11 \mathrm{~A}$ & 0.9700 \\
\hline $\mathrm{C} 5-\mathrm{C} 6$ & $1.394(5)$ & $\mathrm{C} 11-\mathrm{H} 11 \mathrm{~B}$ & 0.9700 \\
\hline $\mathrm{C} 6-\mathrm{C} 7$ & $1.403(5)$ & $\mathrm{C} 13-\mathrm{H} 13$ & 0.9300 \\
\hline $\mathrm{C} 7-\mathrm{C} 8$ & $1.383(6)$ & C14-H14 & 0.9300 \\
\hline $\mathrm{C} 8-\mathrm{C} 9$ & $1.388(6)$ & C15-H15 & 0.9300 \\
\hline $\mathrm{C} 9-\mathrm{C} 10$ & $1.371(6)$ & $\mathrm{C} 16-\mathrm{H} 16$ & 0.9300 \\
\hline $\mathrm{C} 11-\mathrm{C} 12$ & $1.499(6)$ & C17-H17 & 0.9300 \\
\hline $\mathrm{C} 12-\mathrm{C} 17$ & $1.383(6)$ & & \\
\hline $\mathrm{C} 8-\mathrm{O} 2-\mathrm{C} 11$ & $117.4(3)$ & $\mathrm{C} 12-\mathrm{C} 17-\mathrm{C} 16$ & $120.5(5)$ \\
\hline $\mathrm{C} 3-\mathrm{N} 1-\mathrm{C} 4$ & $118.9(4)$ & $\mathrm{C} 2-\mathrm{C} 1-\mathrm{H} 1$ & 120.00 \\
\hline $\mathrm{C} 6-\mathrm{O} 1-\mathrm{H} 1 \mathrm{O}$ & 109.00 & $\mathrm{C} 1 \mathrm{i}-\mathrm{C} 1-\mathrm{H} 1$ & 120.00 \\
\hline $\mathrm{C} 1-\mathrm{C} 1-\mathrm{C} 2$ & $119.8(4)$ & $\mathrm{C} 1-\mathrm{C} 2-\mathrm{H} 2$ & 119.00 \\
\hline $\mathrm{C} 1-\mathrm{C} 2-\mathrm{C} 3$ & $121.3(4)$ & $\mathrm{C} 3-\mathrm{C} 2-\mathrm{H} 2$ & 119.00 \\
\hline $\mathrm{N} 1-\mathrm{C} 3-\mathrm{C} 3^{\mathrm{i}}$ & $118.3(3)$ & $\mathrm{N} 1-\mathrm{C} 4-\mathrm{H} 4$ & $122(2)$ \\
\hline $\mathrm{C} 2-\mathrm{C} 3-\mathrm{C} 3^{\mathrm{i}}$ & $118.9(4)$ & $\mathrm{C} 5-\mathrm{C} 4-\mathrm{H} 4$ & $116(2)$ \\
\hline
\end{tabular}




\begin{tabular}{|c|c|}
\hline $\mathrm{N} 1-\mathrm{C} 3-\mathrm{C} 2$ & $122.8(4)$ \\
\hline $\mathrm{N} 1-\mathrm{C} 4-\mathrm{C} 5$ & $122.2(4)$ \\
\hline $\mathrm{C} 4-\mathrm{C} 5-\mathrm{C} 10$ & $120.5(4)$ \\
\hline $\mathrm{C} 6-\mathrm{C} 5-\mathrm{C} 10$ & $117.7(3)$ \\
\hline $\mathrm{C} 4-\mathrm{C} 5-\mathrm{C} 6$ & $121.8(4)$ \\
\hline $\mathrm{O} 1-\mathrm{C} 6-\mathrm{C} 7$ & $117.5(3)$ \\
\hline $\mathrm{C} 5-\mathrm{C} 6-\mathrm{C} 7$ & $120.7(4)$ \\
\hline $\mathrm{O} 1-\mathrm{C} 6-\mathrm{C} 5$ & $121.8(3)$ \\
\hline $\mathrm{C} 6-\mathrm{C} 7-\mathrm{C} 8$ & $119.6(4)$ \\
\hline $\mathrm{O} 2-\mathrm{C} 8-\mathrm{C} 9$ & $115.0(4)$ \\
\hline $\mathrm{C} 7-\mathrm{C} 8-\mathrm{C} 9$ & $120.5(4)$ \\
\hline $\mathrm{O} 2-\mathrm{C} 8-\mathrm{C} 7$ & $124.5(3)$ \\
\hline $\mathrm{C} 8-\mathrm{C} 9-\mathrm{C} 10$ & $119.2(4)$ \\
\hline $\mathrm{C} 5-\mathrm{C} 10-\mathrm{C} 9$ & $122.3(4)$ \\
\hline $\mathrm{O} 2-\mathrm{C} 11-\mathrm{C} 12$ & $108.6(3)$ \\
\hline $\mathrm{C} 11-\mathrm{C} 12-\mathrm{C} 13$ & $120.9(4)$ \\
\hline $\mathrm{C} 13-\mathrm{C} 12-\mathrm{C} 17$ & $118.8(4)$ \\
\hline $\mathrm{C} 11-\mathrm{C} 12-\mathrm{C} 17$ & $120.3(4)$ \\
\hline $\mathrm{C} 12-\mathrm{C} 13-\mathrm{C} 14$ & $120.8(4)$ \\
\hline $\mathrm{C} 13-\mathrm{C} 14-\mathrm{C} 15$ & $119.8(4)$ \\
\hline $\mathrm{C} 14-\mathrm{C} 15-\mathrm{C} 16$ & $120.0(5)$ \\
\hline $\mathrm{C} 15-\mathrm{C} 16-\mathrm{C} 17$ & $120.2(5)$ \\
\hline $\mathrm{C} 11-\mathrm{O} 2-\mathrm{C} 8-\mathrm{C} 7$ & $1.5(6)$ \\
\hline $\mathrm{C} 11-\mathrm{O} 2-\mathrm{C} 8-\mathrm{C} 9$ & $-177.8(4)$ \\
\hline $\mathrm{C} 8-\mathrm{O} 2-\mathrm{C} 11-\mathrm{C} 12$ & $174.0(3)$ \\
\hline $\mathrm{C} 4-\mathrm{N} 1-\mathrm{C} 3-\mathrm{C} 2$ & $41.3(6)$ \\
\hline $\mathrm{C} 4-\mathrm{N} 1-\mathrm{C} 3-\mathrm{C} 3^{\mathrm{i}}$ & $-139.8(4)$ \\
\hline $\mathrm{C} 3-\mathrm{N} 1-\mathrm{C} 4-\mathrm{C} 5$ & $-176.8(4)$ \\
\hline $\mathrm{C} 1-\mathrm{C} 1-\mathrm{C} 2-\mathrm{C} 3$ & $0.1(8)$ \\
\hline $\mathrm{C} 2-\mathrm{C} 1-\mathrm{C} 1^{\mathrm{i}}-\mathrm{C} 2^{\mathrm{i}}$ & $0.0(9)$ \\
\hline $\mathrm{C} 1-\mathrm{C} 2-\mathrm{C} 3-\mathrm{N} 1$ & $178.9(5)$ \\
\hline $\mathrm{C} 1-\mathrm{C} 2-\mathrm{C} 3-\mathrm{C} 3^{\mathrm{i}}$ & $-0.1(7)$ \\
\hline $\mathrm{N} 1-\mathrm{C} 3-\mathrm{C} 3^{\mathrm{i}}-\mathrm{N} 1^{\mathrm{i}}$ & $0.0(6)$ \\
\hline $\mathrm{N} 1-\mathrm{C} 3-\mathrm{C} 3^{\mathrm{i}}-\mathrm{C} 2^{\mathrm{i}}$ & $-179.0(4)$ \\
\hline $\mathrm{C} 2-\mathrm{C} 3-\mathrm{C} 3^{\mathrm{i}}-\mathrm{N} 1^{\mathrm{i}}$ & $179.0(4)$ \\
\hline $\mathrm{C} 2-\mathrm{C} 3-\mathrm{C} 3^{\mathrm{i}}-\mathrm{C} 2^{\mathrm{i}}$ & $0.0(6)$ \\
\hline $\mathrm{N} 1-\mathrm{C} 4-\mathrm{C} 5-\mathrm{C} 6$ & $-0.9(7)$ \\
\hline $\mathrm{N} 1-\mathrm{C} 4-\mathrm{C} 5-\mathrm{C} 10$ & $-179.5(4)$ \\
\hline $\mathrm{C} 4-\mathrm{C} 5-\mathrm{C} 6-\mathrm{O} 1$ & $2.6(6)$ \\
\hline $\mathrm{C} 4-\mathrm{C} 5-\mathrm{C} 6-\mathrm{C} 7$ & $-178.2(4)$ \\
\hline $\mathrm{C} 10-\mathrm{C} 5-\mathrm{C} 6-\mathrm{O} 1$ & $-178.8(4)$ \\
\hline $\mathrm{C} 10-\mathrm{C} 5-\mathrm{C} 6-\mathrm{C} 7$ & $0.5(6)$ \\
\hline
\end{tabular}

$\begin{array}{ll}\mathrm{C} 6-\mathrm{C} 7-\mathrm{H} 7 & 120.00 \\ \mathrm{C} 8-\mathrm{C} 7-\mathrm{H} 7 & 120.00 \\ \mathrm{C} 8-\mathrm{C} 9-\mathrm{H} 9 & 120.00 \\ \mathrm{C} 10-\mathrm{C} 9-\mathrm{H} 9 & 120.00 \\ \mathrm{C} 5-\mathrm{C} 10-\mathrm{H} 10 & 119.00 \\ \mathrm{C} 9-\mathrm{C} 10-\mathrm{H} 10 & 119.00 \\ \mathrm{O} 2-\mathrm{C} 11-\mathrm{H} 11 \mathrm{~A} & 110.00 \\ \mathrm{O} 2-\mathrm{C} 11-\mathrm{H} 11 \mathrm{~B} & 110.00 \\ \mathrm{C} 12-\mathrm{C} 11-\mathrm{H} 11 \mathrm{~A} & 110.00 \\ \mathrm{C} 12-\mathrm{C} 11-\mathrm{H} 11 \mathrm{~B} & 110.00 \\ \mathrm{H} 11 \mathrm{~A}-\mathrm{C} 11-\mathrm{H} 11 \mathrm{~B} & 108.00 \\ \mathrm{C} 12-\mathrm{C} 13-\mathrm{H} 13 & 120.00 \\ \mathrm{C} 14-\mathrm{C} 13-\mathrm{H} 13 & 120.00 \\ \mathrm{C} 13-\mathrm{C} 14-\mathrm{H} 14 & 120.00 \\ \mathrm{C} 15-\mathrm{C} 14-\mathrm{H} 14 & 120.00 \\ \mathrm{C} 14-\mathrm{C} 15-\mathrm{H} 15 & 120.00 \\ \mathrm{C} 16-\mathrm{C} 15-\mathrm{H} 15 & 120.00 \\ \mathrm{C} 15-\mathrm{C} 16-\mathrm{H} 16 & 120.00 \\ \mathrm{C} 17-\mathrm{C} 16-\mathrm{H} 16 & 120.00 \\ \mathrm{C} 12-\mathrm{C} 17-\mathrm{H} 17 & 120.00 \\ \mathrm{C} 16-\mathrm{C} 17-\mathrm{H} 17 & 120.00\end{array}$

$\mathrm{C} 4-\mathrm{C} 5-\mathrm{C} 10-\mathrm{C} 9 \quad 179.6$ (4)

$\mathrm{C} 6-\mathrm{C} 5-\mathrm{C} 10-\mathrm{C} 9 \quad 0.9(6)$

$\mathrm{O} 1-\mathrm{C} 6-\mathrm{C} 7-\mathrm{C} 8 \quad 177.9(4)$

$\mathrm{C} 5-\mathrm{C} 6-\mathrm{C} 7-\mathrm{C} 8 \quad-1.4(6)$

$\mathrm{C} 6-\mathrm{C} 7-\mathrm{C} 8-\mathrm{O} 2 \quad-178.4(4)$

$\mathrm{C} 6-\mathrm{C} 7-\mathrm{C} 8-\mathrm{C} 9 \quad 0.9(6)$

$\mathrm{O} 2-\mathrm{C} 8-\mathrm{C} 9-\mathrm{C} 10 \quad 179.9(4)$

$\mathrm{C} 7-\mathrm{C} 8-\mathrm{C} 9-\mathrm{C} 10 \quad 0.5(7)$

$\mathrm{C} 8-\mathrm{C} 9-\mathrm{C} 10-\mathrm{C} 5 \quad-1.4(7)$

$\mathrm{O} 2-\mathrm{C} 11-\mathrm{C} 12-\mathrm{C} 13 \quad 96.8(4)$

$\mathrm{O} 2-\mathrm{C} 11-\mathrm{C} 12-\mathrm{C} 17 \quad-84.8(5)$

$\mathrm{C} 11-\mathrm{C} 12-\mathrm{C} 13-\mathrm{C} 14 \quad 176.4$ (4)

$\mathrm{C} 17-\mathrm{C} 12-\mathrm{C} 13-\mathrm{C} 14 \quad-2.0(6)$

$\mathrm{C} 11-\mathrm{C} 12-\mathrm{C} 17-\mathrm{C} 16 \quad-177.4$ (4)

$\mathrm{C} 13-\mathrm{C} 12-\mathrm{C} 17-\mathrm{C} 16 \quad 1.0(7)$

$\mathrm{C} 12-\mathrm{C} 13-\mathrm{C} 14-\mathrm{C} 15 \quad 1.4(7)$

$\mathrm{C} 13-\mathrm{C} 14-\mathrm{C} 15-\mathrm{C} 16 \quad 0.2(7)$

$\mathrm{C} 14-\mathrm{C} 15-\mathrm{C} 16-\mathrm{C} 17 \quad-1.2(8)$

$\mathrm{C} 15-\mathrm{C} 16-\mathrm{C} 17-\mathrm{C} 12 \quad 0.6(8)$

Symmetry code: (i) $-x, y, z$. 
Hydrogen-bond geometry $\left(A,{ }^{\circ}\right)$

$\mathrm{Cg} 2$ is the centroid of the $\mathrm{C} 5-\mathrm{C} 10$ phenol ring.

\begin{tabular}{lllll}
\hline$D-\mathrm{H} \cdots A$ & $D-\mathrm{H}$ & $\mathrm{H} \cdots A$ & $D \cdots A$ & $D-\mathrm{H} \cdots A$ \\
\hline $\mathrm{O} 1-\mathrm{H} 1 O \cdots \mathrm{N} 1$ & 0.82 & 1.90 & $2.622(5)$ & 147 \\
$\mathrm{C} 2-\mathrm{H} 2 \cdots C g 2^{\mathrm{ii}}$ & 0.93 & 2.88 & $3.499(5)$ & 125 \\
$\mathrm{C} 13-\mathrm{H} 13 \cdots C g 2^{\mathrm{iii}}$ & 0.93 & 2.60 & $3.493(5)$ & 161 \\
\hline
\end{tabular}

Symmetry codes: (ii) $x,-y+1, z-1 / 2$; (iii) $x,-y, z+1 / 2$. 